\title{
ATRIOVENTRICULAR CONDUCTION DEFECT ASSOCIATED WITH SEVERE HYPONATREMIA
}

\author{
TURGUT KARABAG, BELMA KALAYCI, MUHAMMET RASIT SAYIN, \\ TURGAY ERTEN
}

\author{
Department of Cardiology, Faculty of Medicine, Bulent Ecevit University, \\ Zonguldak, Turkey
}

\begin{abstract}
Hyponatremia is the most common electrolyte disorder among hospitalized patients and in the clinical setting. Patients with hyponatremia may develop a variety of symptoms, primarily neurological and gastrointestinal. Hyponatremia is more frequently encountered in patients with an underlying heart disease, particularly in the elderly. We hereby present a case of complete atrioventricular block in an elderly patient who had undergone aortic valve replacement and had been using thiazide. Complete atrioventricular block improved after sodium replacement therapy and no other cause of electrolyte disorder was documented.
\end{abstract}

Keywords: hyponatremia, atrioventricular block, thiazide diuretics

\section{Introduction}

Hyponatremia is defined as a serum sodium concentration lower than $135 \mathrm{mmol} / \mathrm{L}$ and is associated with increased mortality, morbidity and prolonged hospitalization [1]. Hyponatremia is mainly caused by medications and inappropriate antidiuretic hormone release2. Patients with hyponatremia may develop a variety of symptoms involving primarily neurological and gastrointestinal systems [2]. Electrolyte disorders are known to affect electrical conduction in the heart [3]. Even though cardiac conduction defects that may be associated with hyponatremia have been described in several case reports and studies, a strong relationship of cause and effect has not been demonstrated yet [4-7]. This study presents a case of complete atrioventricular block in a hyponatremic patient presenting with syncope in whom complete atrioventricular block improved as sodium levels increased and discusses possible mechanisms.

\section{Case report}

A 79-year old female patient was admitted to the emergency service with a 10-day history of complaints of dizziness, nausea, loss of appetite and syncope on the day of admission. The patient had undergone aortic valve replacement (AVR) due to calcific aortic valve stenosis 10 years ago. She reported to have no other chronic diseases

Manuscript received: 10.07.2017

Received in revised form: 25.11.2017

Accepted: 06.12.2017

Address for correspondence: turgutkarabag@yahoo.com but hypertension for 15 years. The patient reported to have been receiving irbesartan $(150 \mathrm{mg})+$ hydrochlorothiazide $(12.5 \mathrm{mg})$ and amlodipine $10 \mathrm{mg}$ for hypertension. In addition, she was receiving warfarin for AVR. On physical examination, the patient with an ongoing dizziness had blood pressure of $120 / 75 \mathrm{mmHg}$, heart rate of $41 / \mathrm{min}$ and body temperature of $36.6^{\circ} \mathrm{C}$. A cardiac examination revealed prosthetic aortic valve sound and 2/6 systolic murmur. Other system examinations revealed no abnormalities. Neurological examination was unremarkable. There was minimal edema in both legs. Teleradiography revealed no pathology. Her electrocardiogram showed bradycardic heart rate $(41 / \mathrm{min})$, right bundle branch block and 2:1 atrioventricular (AV) block (Figure 1). Laboratory tests revealed sodium level of $104 \mathrm{mmol} / \mathrm{L}(132-146)$, potassium of $4.2 \mathrm{mmol} / \mathrm{L}(3.5-5.5)$, urea of $53 \mathrm{mg} / \mathrm{dL}(0-50)$, creatinine of $1.3 \mathrm{mg} / \mathrm{dL}(0-1.2)$, calcium of $9.1 \mathrm{mg} / \mathrm{dL}(8.8-10.6)$, and magnesium of $1.6 \mathrm{mg} /$ $\mathrm{dL}$ (1.8-2.6). International normalised ratio was 2.55. Cardiac markers were normal. On echocardiography and fluoroscopy, prosthetic aortic valve opening was normal. Gradient across the valve was normal with a maximum $14 \mathrm{mmHg}$ and mean $7 \mathrm{~mm} \mathrm{Hg}$. There was mild mitral and tricuspid regurgitation. Pulmonary systolic pressure across the tricuspid valve was $35 \mathrm{~mm} \mathrm{Hg}$. The ejection fraction was normal. Cranial tomography was unremarkable.

The patient was transferred to the coronary intensive care unit with the diagnosis of hyponatremia and 2:1 AV block. Since the patient was hemodynamically stable, the insertion of a pacemaker was deemed unneccessary. 
Antihypertensive drugs the patient was using, which might have caused hyponatremia, were discontinued. Serum uric acid was $5.2 \mathrm{mg} / \mathrm{dL}$. Plasma osmolality was low with a 232 $\mathrm{mOsm} / \mathrm{kg}$. The urine osmolality and the urine sodium were reduced $(178 \mathrm{mmol} / \mathrm{kg}$ and $<12 \mathrm{mmol} / \mathrm{L}$, respectively). Renal and liver functions, thyroid function tests and morning cortisol level were all normal. The case was consulted with the internal medicine department and it was decided to initiate the patient on $3 \% \mathrm{NaCI}$ hypertonic saline. Patient's 24-hour sodium level, 24-hour creatinine and creatinine clearance were all normal. The heart rate of the patient returned to normal with a intermittent 2:1 AV block on day 2 (Figure 2).
The sodium level was $110 \mathrm{mmol} / \mathrm{L}$. On day 3 , the sodium level was $122 \mathrm{mmol} / \mathrm{L}$ and first degree AV block with a $220 \mathrm{msec}$ PR interval was developed on ECG (Figure 3). Normal sinus rhythm was noted on day 4 with a $126 \mathrm{mmol} / \mathrm{L}$ sodium value (Figure 4). Patient's complaints completely resolved. Detailed examinations showed normal levels of adrenocorticotropic hormone and growth hormone. Holter recording showed no AV block. A total of 1050 ventricular extrasystoles were recorded in 24 hours. The patient was discharged with irbesartan $300 \mathrm{mg}$ and amlodipine $10 \mathrm{mg}$. First, third and 6th month examinations and holter tests revealed no further conduction defects. The patient had no complaints.

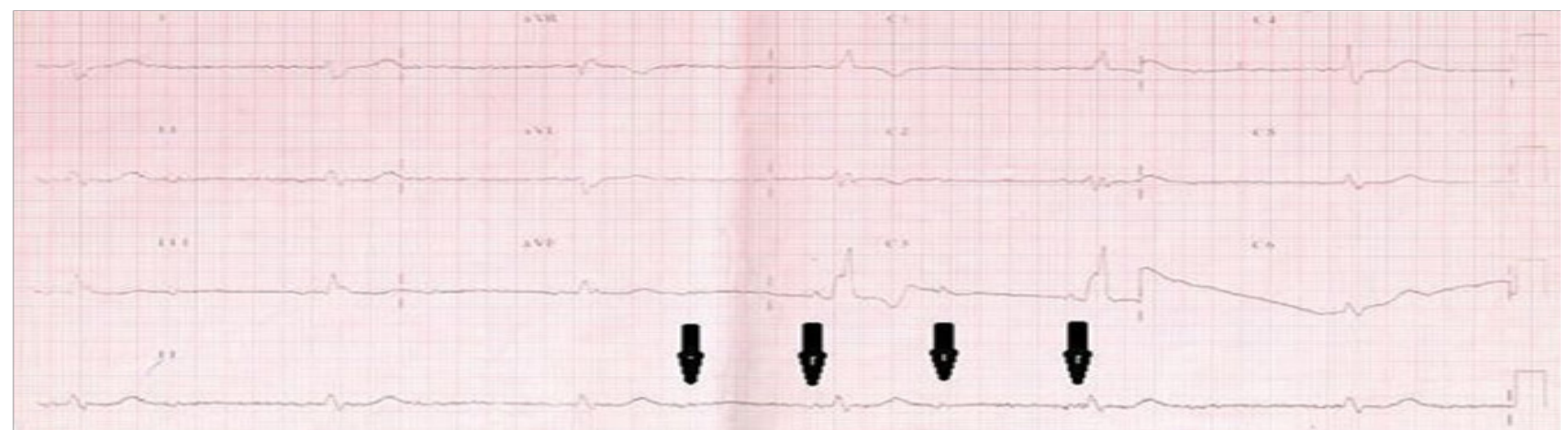

Figure 1. Electrocardiography showing bradycardia and $2: 1$ atrioventricular block with a 104 mmol/L sodium level at day 1 . (arrows indicate $\mathrm{P}$ waves).

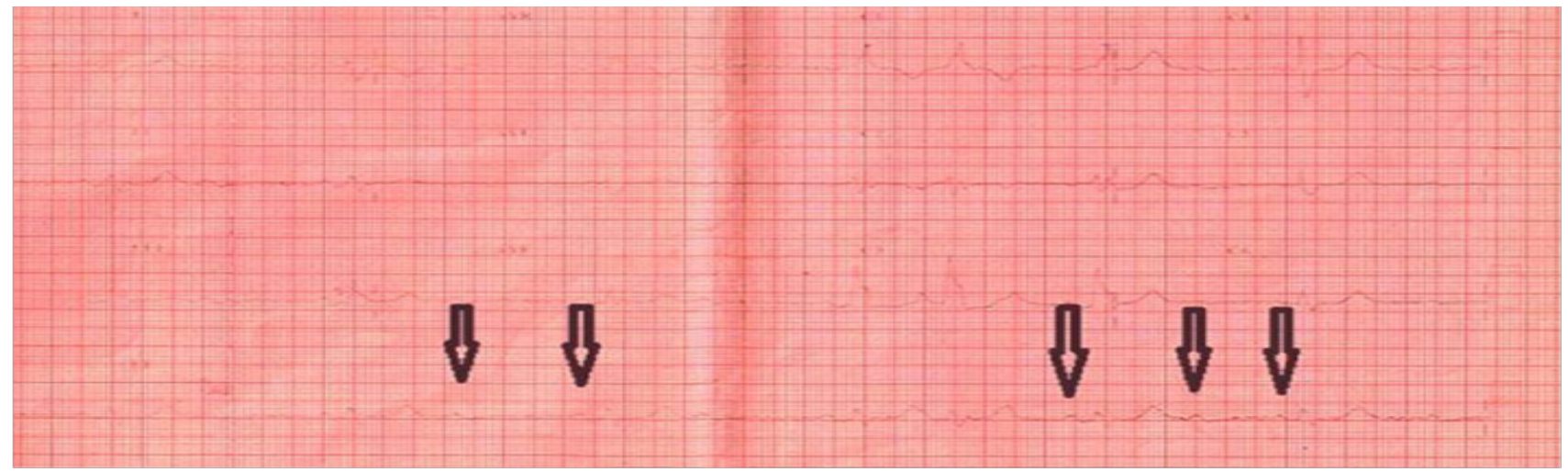

Figure 2. Electrocardiography showing normal heart rate and intermittent 2:1 atrioventricular block with a $110 \mathrm{mmol} / \mathrm{L}$ sodium level at day 2. (arrows indicate $\mathrm{P}$ waves).

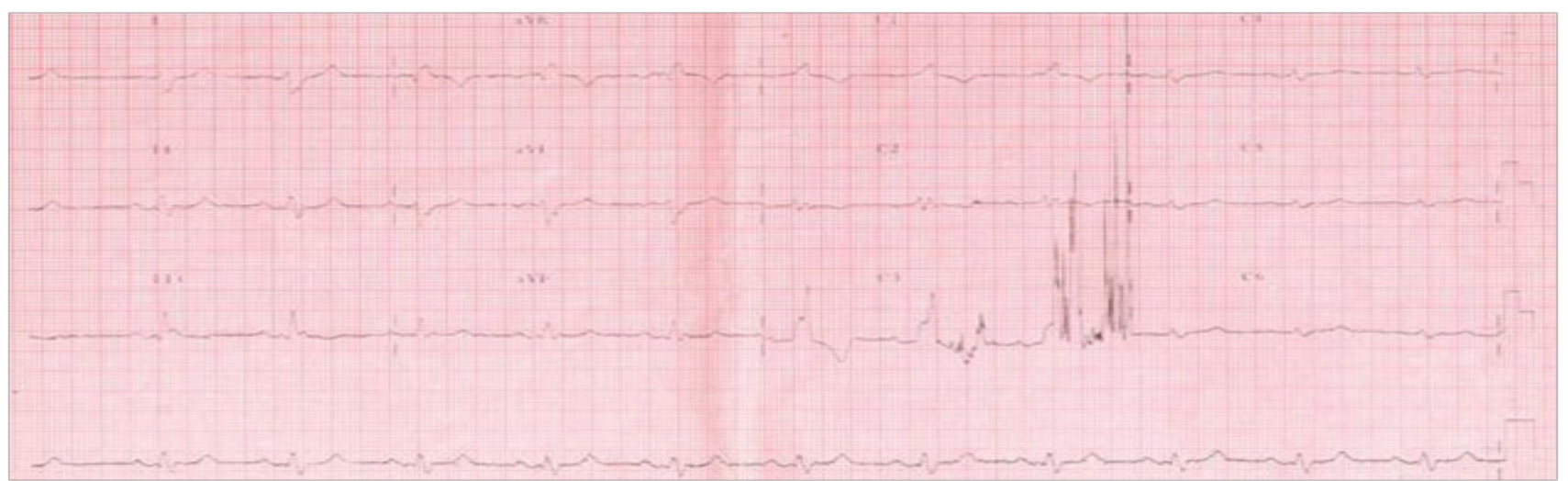

Figure 3. Electrocardiography showing first degree AV block with a $220 \mathrm{msec}$ PR interval at day 3 (Sodium level was $122 \mathrm{mmol} / \mathrm{L}$ ). 


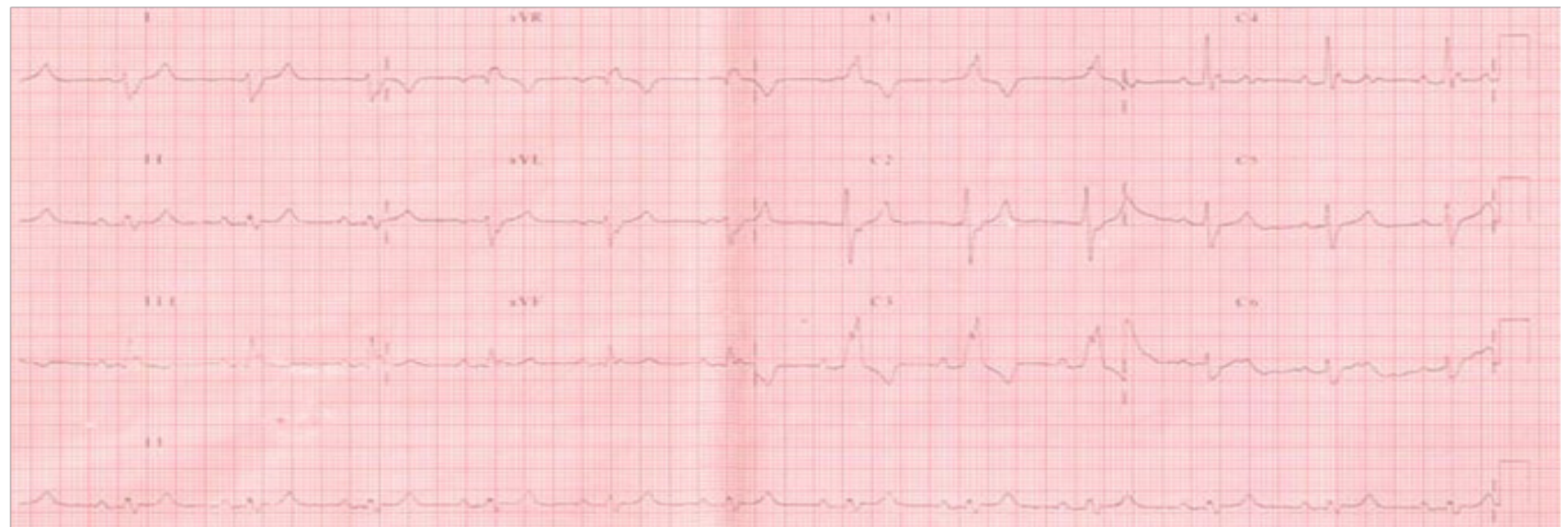

Figure 4. Normal sinus rhythm recorded at day 4 (sodium level was $126 \mathrm{mmol} / \mathrm{L}$ ).

\section{Discussion}

Hyponatremia is the most common electrolyte abnormality among hospitalized patients and is also a common clinical condition among hospitalized heart failure patients. Since patients with hyponatremia may develop brain edema due to low sodium levels, various neurological and gastrointestinal symptoms can be encountered [8]. There are few studies addressing hyponatremia in the literature. Conduction defects, which are primarily associated with hyponatremia, may be accompanied by several clinical conditions including primary cardiac disorder, antiarrhythmic drug use and other electrolyte abnormalities [7]. A study by Jeong et al, reported the first case of complete atrioventricular block in an elderly woman who had also been taking thiazide [4]. In a review by Liamis et al, it was suggested that thiazide use was a primary cause of hyponatremia and might result in significant complications in elderly patients [8]. In the case presented here, the patient was elderly and had been taking an antihypertensive containing thiazide. No other causes of hyponatremia could be identified and no hyponatremia or atrioventricular block occurred after the cessation of thiazide at the follow-ups, which indicates that the conduction defect of the patient was associated with hyponatremia, and hyponatremia was associated with thiazide use. Although the potassium levels were normal, normal uric acid level, reduced plasma osmolality, urine osmolality and urine sodium were lower which were consistent with thiazide-associated hyponatremia.

Thiazide-associated hyponatremia is observed mainly in patients with certain risk factors such as those receiving large doses of thiazides, having several comorbidities (heart failure, liver disease or malignancy) [8]. Our patient was hypertensive and had undergone aortic valve replacement. However she did not have any more chronic disease. A variety of drug categories that elderly patients frequently take, such as non-steroidal anti-inflammatory drugs, selective serotonin re-uptake inhibitors ,tricyclic antidepressants, benzodiazepins, angiotensin converting enzyme inhibitors, sartans, impair water excretion [8]. Our patient was on combination of AT-1 receptor antagonist and a low dose of hydrochlorothiazide (12.5 mg). This combination may have caused adverse effects in the development of hyponatremia.

On the other hand, the presence of preexisting heart disease in the patient is likely to be a precipitating factor for the conduction defect. The relationship between hyponatremia and conduction defect is yet to be elucidated. Several empirical studies have proposed various theories in this regard. In theory, the decrease of extracellular sodium levels can reduce inward sodium flow at the initial phase of action potential onset [7]. An empirical study by Trautwein et al showed that extremely low levels of sodium in the fluid perfusing the isolated heart muscle reduced the number of contractions as well as excitability and conduction velocity [8]. A study on rabbit hearts by Nikolaidou et al demonstrated that low sodium levels could affect action potentials in the AV node [10]. It has also been shown that the decrease of extracellular sodium in cell culture reduces inward sodium flow and action potentials [11].

In the case presented here, the patient was elderly and had undergone an aortic valve replacement previously. We believe that the patient developed complete atrioventricular block due to hyponatremia caused by the use of an antihypertensive containing thiazide. This case presented here is a rare example where the patient developed advanced conduction defect due to hyponatremia. As the patient's sodium levels returned to normal, conduction defect also improved and no further conduction defects were identified at follow-ups, which made us associate temporary conduction defect with low sodium levels.

In conclusion, hyponatremia occurs more commonly and easily in elderly patients taking thiazide. These patients can develop various conduction defects. One should be alert to signs of possible conduction defects particularly in patients who have conditions that are likely to induce 
hyponatremia and who are on medications. Hyponatremic patients should be questioned about conduction defects and associated symptoms and hospitalized patients should be closely monitored.

\section{References}

1. Tang Q, Hua Q. Relationship between hyponatremia and in-hospital outcomes in Chinese patients with ST-elevation myocardial infarction. Intern Med. 2011;50:969-974.

2. Goh KP. Management of hyponatremia. Am Fam Physician. 2004; 15;69(10):2387-2394.

3. Tse G, Yeo JM. Conduction abnormalities and ventricular arrhythmogenesis: The roles of sodium channels and gap junctions. Int J Cardiol Heart Vasc. 2015;9:75-82.

4. Jeong JJ, Kim DH, Yoo SI, Shin SH, Lee MD, Ye JH, et al. Complete atrioventricular block associated with hyponatremia. Korean J Med. 2012;83(1):97-100.

5. Suri PHK, Alai MS, Rather AH, Jalal S. Hyponatermia presenting as cardiac conduction defect. JK Sci. 2009;11(2):85-86.

6. Kottwitz J, Akdis D, Duru F, Heidecker B. Severe Hyponatremia
Leading to Complete Atrioventricular Block. Am J Med. 2016;129:e243-e244.

7. Mouallem M, Friedman E, Shemesh Y, Mayan H, Pauzner R, Farfel Z. Cardiac conduction defects associated with hyponatremia. Clin Cardiol. 1991;14:165-168.

8. Liamis G, Filippatos TD, Elisaf MS. Thiazide-associated hyponatremia in the elderly: what the clinician needs to know. J Geriatr Cardiol. 2016;13:175-182.

9. Trautwein W. Generation and conduction of impulses in the heart as affected by drugs. Pharmacol Rev. 1963;15,277-332.

10. Nikolaidou T, Cai XJ, Stephenson RS, Yanni J, Lowe T, Atkinson AJ, et al. Congestive Heart Failure Leads to Prolongation of the PR interval and atrioventricular Junction Enlargement and Ion Channel Remodelling in the Rabbit. PLoS One. 2015 Oct 28;10(10):e0141452. doi: 10.1371/journal.pone.0141452.

11. Lu YY, Cheng CC, Chen YC, Lin YK, Chen SA, Chen YJ. Electrolyte disturbances differentially regulate sinoatrial node and pulmonary vein electrical activity: A contribution to hypokalemia- or hyponatremia-induced atrial fibrillation. Heart Rhythm. 2016;13(3):781-788. 